\title{
Sentido y Propósito de la Violencia Escolar. Análisis Estructural del Discurso de Estudiantes Agresivos del Nivel Socioeconómico Alto ${ }^{1}$
}

\section{Mesaning and Purpose of School Violence: Structural Analysis of Discourse of aggressive students of high socio-economic status.}

\author{
Mauricio García Phd.* \\ Pablo Madriaza Ps.**
}

\begin{abstract}
Resumen
Frecuentemente la violencia es asociada con los sectores más desposeídos de la sociedad. La violencia escolar no escapa a estas representaciones: suele asociarse a barrios marginales y escuelas pobres, dejando fuera de la comprensión, muchas de las formas violentas que emergen en otros segmentos socioeconómicos, especialmente los más acomodados. No obstante los recientes hechos en nuestro país, dan muestras suficientes para abordar el tema desde una perspectiva comprensiva. Se puede pensar fácilmente en una violencia del individuo marginal y pobre, como una violencia contestaría; no obstante, esta idea no es fácil de llevar al contexto de las clases sociales más pudientes. Si en estas clases sociales, la realidad material no se plantea como un problema, entonces la pregunta acerca del sentido de la violencia escolar se plantea aún con más fuerza. La siguiente investigación es el resultado del análisis cualitativo estructural del discurso de un grupo de jóvenes de nivel socioeconómico alto de enseñanza media, que fueron considerados por sus instituciones educativas como violentos o agresivos.
\end{abstract}

Palabras claves: violencia escolar, sentido de la violencia, violencia escolar en NSE alto.

1 Este artículo corresponde a un aspecto parcial de los resultados del Proyecto Fondecyt No $1010771:$ "Hacia una Interpretación de la Violencia en la Escuela. Lineamientos de Estrategias de Convivencia Social en el Espacio Educativo". Aprobado para el año 2001. Además se inserta en el marco del Proyecto Fondecyt $\mathrm{N}^{\mathrm{O}}$ 1040694: "Figuras Estructurales de la Violencia Escolar. Hacia una Recuperación de la "Subjetividad" Educati$v a^{\prime \prime}$. Aprobado para el año 2004.

* Mauricio García, Psicoanalista, Doctor en Psicología de la U.C. de Lovaina. Docente Escuela de Psicología de la Universidad Alberto Hurtado y Escuela de Psicología de la PUC.mgarcia@uahurtado.cl.

** Psicólogo Pontificia Universidad Católica de Chile, Magíster (C) en Antropología y Desarrollo de la Universidad de Chile.pmadriaz@puc.cl 


\begin{abstract}
Violence is frequently associated with economically deprived populations of society. School violence does not escape from these representations: usually, it is associated to low income neighborhoods and schools, not acknowledging and understanding several violent manifestations that emerge in other socio economic groups, particularly the higher ones. Nevertheless, recent events in our country have shown enough evidence to sustain a more comprehensive approach to the matter. It is easy to think of violence in poor and marginal individuals as a reactive one. This notion is not easily translated in to the higher socio-economic levels so that the question about the meaning of school violence in this population rises strongly. The present study constitutes the outcome of a structural qualitative analysis of the discourse of a group of high-school students of high socio economic status, who where considered by their educational institutions as violent or aggressive.
\end{abstract}

Key words: School violence, meaning of violence, school violence in high socio economic status groups.

\section{Introducción}

La violencia parece ser una permanente fuente de preocupación para las sociedades contemporáneas. Frecuentemente los pensadores e investigadores occidentales han orientado sus preguntas hacia las causas de la violencia. Aún hoy esta pregunta parece reinstalarse una y otra vez, a propósito de las diversas formas de violencia que se van diferenciando, dependiendo de los contextos en los cuales va emergiendo.

Una de estas violencias -que recientemente se ha visibilizado como tal, para el mundo de las ciencias sociales-, ha sido la violencia escolar. Mucho se ha escrito acerca de los determinantes y características que tendrían este tipo de relaciones violentas, así como cuáles serían sus actores principales. Sin embargo, es frecuente asociar cada una de estas formas de violencia social a un sujeto particular - principalmente marginal y pobre- que sería el principal actor de ésta. Foucault plantea por ejemplo -en relación al delincuente-, que las tecnologías sociales incluso pueden llegar a describir las características físicas que tendrían estos individuos (1976). La comprensión disciplinar y social sobre la violencia escolar no escapa a estos imaginarios sociales. De esta forma, aquella violencia de la "elite" social queda invisibilizada bajo el alero de la violencia marginal. Sin embargo, algunos investigadores rechazan esta supuesta brecha que habría entre los dos polos económicos, y plantean que esta diferencia es mucho más atenuada de lo que se podría creer (Navarro, 2003).

Se puede pensar fácilmente en una violencia del individuo marginal y pobre, como una violencia contestaría (Dubet, 1998; Magenzo \& Donoso, 2000); no obstante, esta idea no es fácil de llevar al contexto de las clases sociales más pudientes. Si en estas clases sociales, la realidad material no se plantea como un problema, entonces la pregunta acerca del sentido de la violencia escolar en estos contextos, se plantea aún con más fuerza.

El contexto en que emerge este artículo, justamente intenta tratar de describir desde una mirada psico-antropológica, el sentido de la violencia escolar para jóvenes provenientes de los sectores más pudientes de la sociedad chilena. Es un intento, por tratar de hacer emerger desde el discurso de los propios actores, las relaciones simbólicas, los contextos, motivos y sentidos que adquiere el ser violento en un mundo urbano y aparentemente satisfecho. 


\section{Antecedentes}

Las primeras investigaciones sistemáticas en el mundo comenzaron a finales de los años sesenta a cargo de un grupo de investigadores nórdicos que confeccionaron el primer cuestionario para dimensionar el problema (Ortega \& Mora-Merchán, 1997; Olweus, 1998). De ahí en adelante la preocupación y emergencia de este fenómeno ha ido creciendo paulatinamente. No obstante una gran dificultad a la hora de comparar los datos mundiales, ha sido las múltiples definiciones con las que cuenta la violencia escolar. Olweus pone el acento en la intimidación y el acoso: "un alumno es agredido o se convierte en victima cuando está expuesto, de forma repetida y durante un tiempo, a acciones negativas que lleva a cabo otro alumno o varios de ellos" (Olweus, 1998, pp 25).

Teniendo en cuenta que los estudios usan definiciones distintas, la prevalencia del fenómeno en diversos países tiende a rondar entre el 10 y el $15 \%$ (National Center for Education Statistics, 2002; Olweus, 1998; Ortega \& Mora-Merchán, 1997; Whitney \& Smith, 1993; Wordes \& Nunez, 2002).

Latinoamérica y Chile. Inicialmente se aborda el problema a través de la interpretación de la violencia social en general usando modelos provenientes de la investigación en violencia intrafamiliar. Posteriormente aparecen algunos estudios que arrojan indicadores más específicos de violencia escolar. En Brasil el $13 \%$ de los estudiantes reportó que estudiantes y profesores usan armas al interior de la escuela (Abramovay, 2003). En Uruguay la percepción de violencia parece estar más acentuada: el $28 \%$ de los estudiantes reconoce haber participado en peleas y el $71 \%$ dice haber sido agredido verbalmente (Viscardi, 2003). En Ecuador el $26 \%$ de los niños entre 6 y 10 años reconoce haber participado en una pelea y "el $36.7 \%$ de los adolescentes de 11 a 13 años han recibido y proporcionado golpes varias veces" (Maluf, Cevallos \& Cordoba, 2003, pp 281). Con todo, la influencia de los modelos de la violencia intrafamiliar ha implicado que la distinciones entre victimas y agresores haya ocupado un lugar central, lo mismo que las concepciones en torno al abuso y al maltrato (Contador, 2003; Mena \& Vizcarra, 2003).

Otro enfoque, inspirado en Dubet, entiende la violencia escolar como "violencia de la escuela" .Se incluyen por tanto no solo las relaciones interpersonales y sus formas abusivas, sino también el factor institucional y social en la caracterización de fenómeno. De ahí, que la idea de conflicto entre la reproducción cultural a través de la institución educacional (Bordieu, 1977) y la resistencia con la que se opone el alumnado, parece ser fundamental para entender que la escuela en sí misma también es productora de violencia (Llaña, 2002; Magenzo \& Donoso, 2000).

En Chile, como plantean Navarro (2003) y Zeron (2004), el problema de la violencia escolar apenas se empieza a vislumbrar desde mediados de los años 90, teniendo casi todos estos estudios un corte fundamentalmente cualitativo y exploratorio, y observándose una fuerte ausencia de estadísticas nacionales con las que se pueda sopesar la magnitud del fenómeno.

A partir de los datos de las cuatro encuestas que ha hecho el Instituto Nacional de la Juventud, puede observarse un progresivo aumento en la percepción de que la violencia física entre los estudiantes es uno de los problemas frecuentes al interior de los establecimientos educacionales (2002). En su última versión, el 40,3\% de los estudiantes percibe este problema como relevante (INJUV, 2002). No obstante esta percepción progresiva puede deberse a la también progresiva importancia mediática que ha tenido este fenómeno en los últimos años. En un estudio sobre violencia escolar en la Región Metropolitana, se constató que el 15\% de estudiantes de enseñanza media refiere haber llevado cuchillos al colegio y $5 \%$, refiere haber llevado otro tipo de armas. Entre las variables asociadas, se encontró una progresi- 
va disminución de la percepción de violencia, a medida que aumentaban el nivel del curso (Contador, 2003). En otro estudio, esta vez hecho sobre el grupo $5^{\circ}$ a $8^{\circ}$ básico, se pudo observar que el $11,3 \%$ de estos dice haber sido agredido por un compañero más de cinco veces y el 10,3\% confiesa haber agredido en la misma cantidad a otro alumno (López \& Filsecker, 2001).

Pese a la parcialidad de la estadística, los porcentajes tanto en Latinoamérica como en Chile, suelen rondar los márgenes que se observan en países desarrollados de Europa y América.

Fuera de esta comprensión cuantitativa del fenómeno, la idea de indagar sobre el sentido que tendría esta violencia en las escuelas es poco frecuente en el mundo, sobre todo si se interroga el sentido desde el discurso de aquellos que frecuentemente participan de estos actos y que se entienden como agresores. La violencia en cualquiera de sus formas tiende a instalarse como un sinsentido, ubicándose preferentemente en un lugar de exclusión de lo social. En Chile es frecuente el uso de la categoría "antisocial", para cualquier individuo que haga uso de la violencia o de la delincuencia. Es decir, la violencia parece no tener nada que ver con lo social y parece ubicarse al límite de ésta. La violencia -propone Luhmann-, es aquella presencia constantemente ausente en el poder (1991). De ahí se entiende el aborrecimiento que las sociedades contemporáneas han hecho de ésta (Lipovetski, 2000). No obstante la violencia parece estar mucho más ligada al campo de lo social de lo que el imaginario del sentido común pretende. Los antropólogos desde bastante tiempo han propuesto que la violencia se encuentra en muchas de las formas primitivas de lo social, y que al encontrarse en sus inicios civilizatorios, dan cuenta de muchas de las formas sociales actuales (Mauss, 1995; Girard, 1983; Clastres, 2004).

En algunos artículos ya publicados (Madriaza \& García 2004a, 2004b), proponemos que la violencia escolar, parece estar imbricada directamente con la formación de nuevos grupos socioculturales y con una suerte de estructuración espontánea de las relaciones sociales, a falta de un ordenamiento claro para los jóvenes.

\section{Método}

\section{Participantes}

Desde una muestra original compuesta por $64^{2}$ estudiantes se seleccionaron 7 casos cuyo material era "denso" (Geertz, 1986), todos hombres, de tres establecimientos educacionales, que provenían del sector socioeconómico alto de la sociedad y que cumplían con el criterio de haber sido señalados por su institución educativa y / o sus compañeros como agresivos o violentos; y que del mismo modo, habían participado en hechos de violencia en su escuela.

\section{Técnica de Recolección de Datos}

La información fue recolectada por medio de la entrevista comprensiva, cuyo foco es tratar de vislumbrar el sistema de valores, elecciones sociales y lingüísticas que hace el informante y de adherir (sin exceso), con el fin de "liberar" el discurso y reforzar su sinceridad (Kaufmann, 1997).

2 La muestra original seleccionada fueron 64 estudiantes de Enseñanza Media de Chile de 11 establecimientos educacionales del país. La distribución por sexo fue de 12 mujeres y 52 hombres. La edad fluctuaba entre los 14 y 21 años, y se distribuían entre los diversos niveles de enseñanza ( $1^{\circ}$ a $4^{\circ}$ medio). De estos, 24 estudiantes correspondían a 4 colegios de NSE Bajo; 20 estudiantes correspondían a 3 colegios de NSE Alto y 20 estudiantes correspondían a 4 colegios de NSE Medio. 


\section{Procedimiento}

A partir de una invitación extensiva a participar de la investigación, que se les hizo a diversos colegios preseleccionados por las características expuestas más arriba, se prefirió en primera instancia, a aquellos que fueran más representativos de los diferentes niveles socioeconómicos.

Con esta preselección, se inició la búsqueda de informantes que cumplieran con los requisitos expuestos. Esto se hizo, a través de entrevistas preliminares con directivos $y$ docentes que propusieron una lista de posibles informantes, lo cual fue corroborado para la muestra final, con otros pares o a través de estos mismos estudiantes previamente seleccionados.

En la primera fase, se realizaron variadas entrevistas individuales, donde se descartaron muchos de los informantes por no corresponder a los criterios del estudio. En algunos casos, se tuvo que buscar en otros establecimientos nuevamente, siguiendo el procedimiento antes expuesto hasta llegar a aquellos más significativos.

En base al material de las entrevistas, se realizaron posteriormente entrevistas grupales, donde se congregó a un número significativo de estudiantes. Así se llegó a completar 54 entrevistas individuales y 27 grupos focales.

Con el material recogido y transcrito, se realizó el análisis preliminar de todo el material, por medio del modelo de análisis cualitativo de la Grounded Theory (Strauss \& Corbin, 1994) 3 .
Pero dado que tiene interés entender este fenómeno en el NSE alto, -por la escasa investigación al respecto-, quisimos realizar un análisis específico en este grupo. Para ello se seleccionaron las entrevistas individuales que correspondían a este $\mathrm{NSE}^{4}$ y se procedió a realizar un segundo método de análisis. Esta vez se utilizó el análisis estructural de discurso. Según este tipo de análisis, el discurso se presenta a través de oposiciones que reflejan valoraciones antagónicas (positivas y negativas) (Piret, Nizet \& Bourgeois, 1996). A través de esta presunción, se persigue comprender correctamente o atribuir a lo que dice el informante el sentido que efectivamente pone en el discurso. De esta forma, lo relevante es resaltar las relaciones entre sus elementos, más allá de su frecuencia u orden de aparición en el texto (Piret et al, 1996). Se optó por cambiar el tipo de análisis, pues justamente este tipo de método permite resaltar ante todo, el sentido o cierta direccionalidad del discurso. A través de este análisis se desarrollaron dos de las estructuras que el modelo propone para representar la información: la estructura paralela y una forma simplificada de la estructura actancial.

En el establecimiento de las categorías de análisis (ejes semánticos) nos resultó de particular utilidad el modelo propuesto por Jean Pierre Hiernaux (1995), cuyos elementos explicaremos con más atención más adelante.

Estas estructuras resultantes del análisis fueron cotejadas de manera independiente por dos expertos en análisis estructural en dos tiempos distintos ${ }^{5}$. El primero revisó los análisis iniciales que conformaron el modelo y el segundo cotejó el análisis final ya establecido.

3 Este análisis preliminar, fue realizado por los mismos entrevistadores, esta vez en parejas, supervisados por un coordinador general. De esta forma, se codificó los datos a través de tres instancias propuestas por el modelo: codificación abierta, axial y selectiva. En un segundo momento, el investigador principal y el coordinador general, revisaron y cotejaron este análisis preliminar con el material de las entrevistas y Grupos focales, como un modo de triangular y establecer el análisis final de las codificaciones.

4 Dada la naturaleza del análisis estructural se decidió privilegiar el análisis de las entrevistas individuales de este NSE, dejando de lado por ello los grupos focales.

5 Agrademos a los profesores Fernando De Laire Phd. y Alvaro Salinas Phd. por sus aportes en la corrección de estos análisis. 


\section{Resultados}

1. Esquemas de las estructuras de seis informantes

A continuación exponemos los esquemas de análisis de seis casos. Lamentablemente por razones de espacio no podemos exponer la paráfrasis de todos modelos, pero el lector podrá hacerla siguiendo la misma lógica de la paráfrasis de los dos primeros casos desarrollados más abajo.

Figura 1. Estructuras discursi vas de informantes

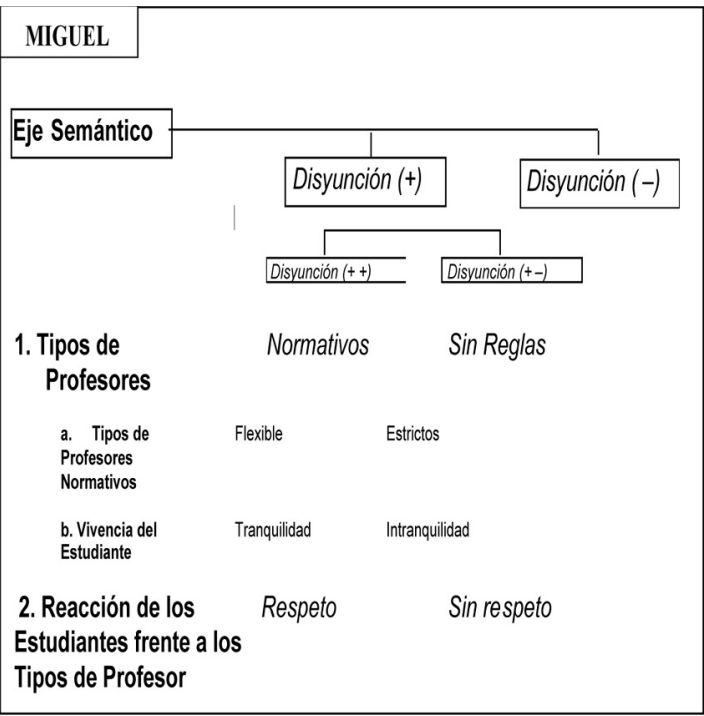

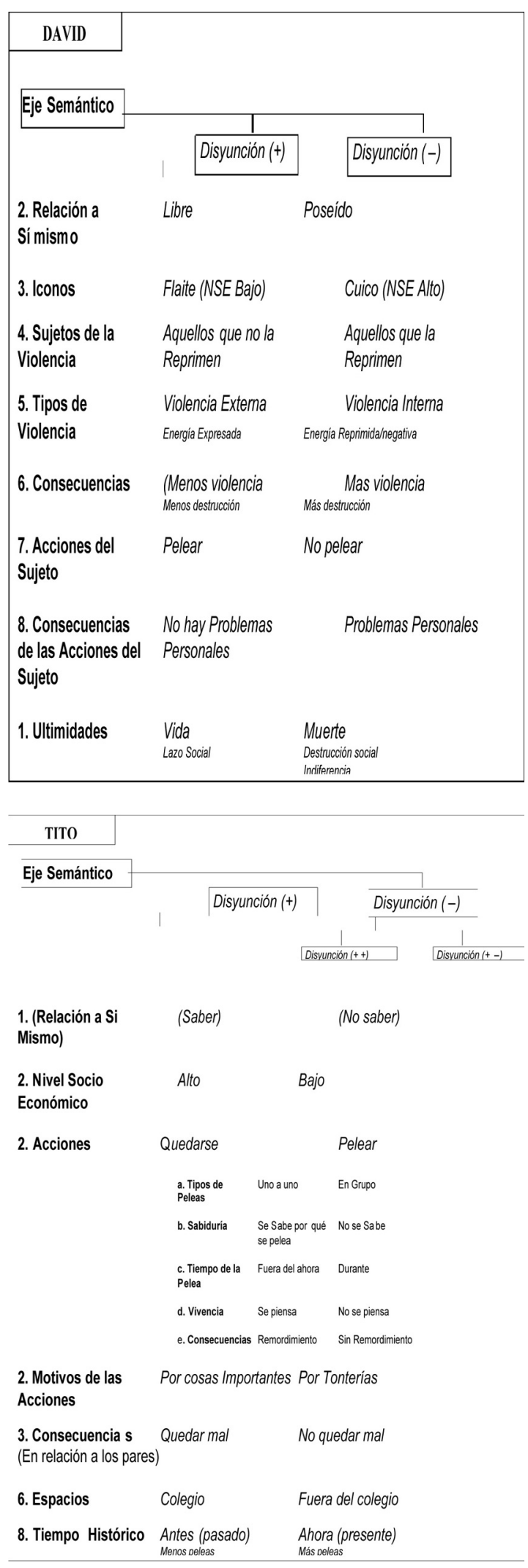




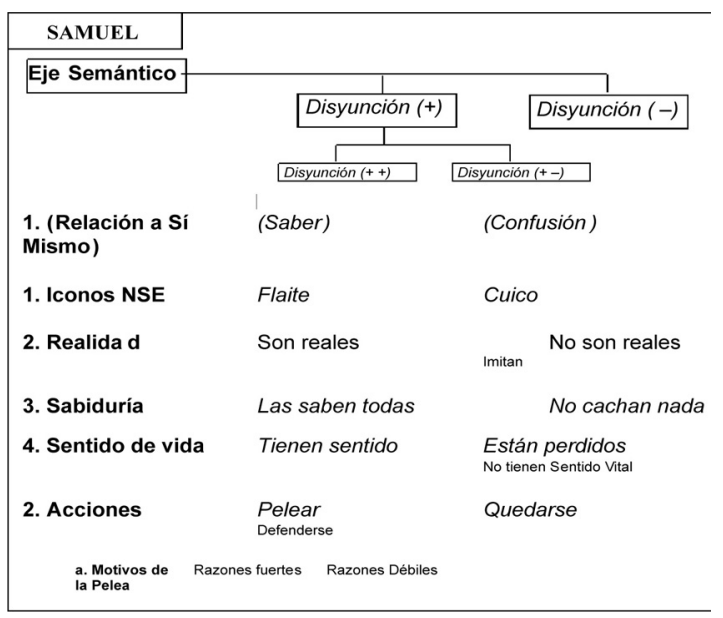

Figura 2. Estructuras discursi vas de informantes.

\begin{tabular}{|c|c|c|}
\hline LALO & & \\
\hline \multirow[t]{2}{*}{ Eje Semántico } & & \\
\hline & Disyunción & \begin{tabular}{|l|} 
Disyunción (- \\
\end{tabular} \\
\hline $\begin{array}{l}\text { 1. (Relación a Sí } \\
\text { Mismo) }\end{array}$ & (Olvidar) & (Rememorar) \\
\hline $\begin{array}{l}\text { 2. Temporalidad de } \\
\text { los Sujetos }\end{array}$ & Ahora & Fuera del Ahora \\
\hline 3. Acciones & Pelea & Quedarse \\
\hline $\begin{array}{l}\text { 4. Modos en que } \\
\text { se vive el Tiempo }\end{array}$ & $\begin{array}{l}\text { Intemporalidad } \\
\text { No se piensa } \\
\text { Sin arrepentimiento }\end{array}$ & $\begin{array}{l}\text { Temporalidad } \\
\text { Arrepentimiento }\end{array}$ \\
\hline $\begin{array}{l}\text { 5. Consecuencias } \\
\text { de la pelea }\end{array}$ & $\begin{array}{l}\text { Tranquilidad } \\
\text { Sin Rabia }\end{array}$ & $\begin{array}{l}\text { Intranquilidad } \\
\text { Irritable }\end{array}$ \\
\hline $\begin{array}{l}\text { 6. Genero de los } \\
\text { Actores }\end{array}$ & Hombre & Mujer \\
\hline $\begin{array}{l}\text { 7. Consecuenci as de } \\
\text { La pelear (por genero) }\end{array}$ & Olvido & Rememoración \\
\hline
\end{tabular}

\begin{tabular}{|c|c|c|}
\hline LALO & & \\
\hline \multirow[t]{2}{*}{ Eje Semántico } & & \\
\hline & Disyunción & \begin{tabular}{|l|} 
Disyunción (- \\
\end{tabular} \\
\hline $\begin{array}{l}\text { 1. (Relación a Sí } \\
\text { Mismo) }\end{array}$ & (Olvidar) & (Rememorar) \\
\hline $\begin{array}{l}\text { 2. Temporalidad de } \\
\text { los Sujetos }\end{array}$ & Ahora & Fuera del Ahora \\
\hline 3. Acciones & Pelea & Quedarse \\
\hline $\begin{array}{l}\text { 4. Modos en que } \\
\text { se vive el Tiempo }\end{array}$ & $\begin{array}{l}\text { Intemporalidad } \\
\text { No se piensa } \\
\text { Sin arrepentimiento }\end{array}$ & $\begin{array}{l}\text { Temporalidad } \\
\text { Arrepentimiento }\end{array}$ \\
\hline $\begin{array}{l}\text { 5. Consecuencias } \\
\text { de la pelea }\end{array}$ & $\begin{array}{l}\text { Tranquilidad } \\
\text { Sin Rabia }\end{array}$ & $\begin{array}{l}\text { Intranquilidad } \\
\text { Irritable }\end{array}$ \\
\hline $\begin{array}{l}\text { 6. Genero de los } \\
\text { Actores }\end{array}$ & Hombre & Mujer \\
\hline $\begin{array}{l}\text { 7. Consecuenci as de } \\
\text { La pelear (por genero) }\end{array}$ & Olvido & Rememoración \\
\hline
\end{tabular}

\begin{tabular}{|l|l|}
\hline \multicolumn{1}{|c|}{ PEDRO } & \\
\hline Eje Semántico & \\
\hline
\end{tabular}




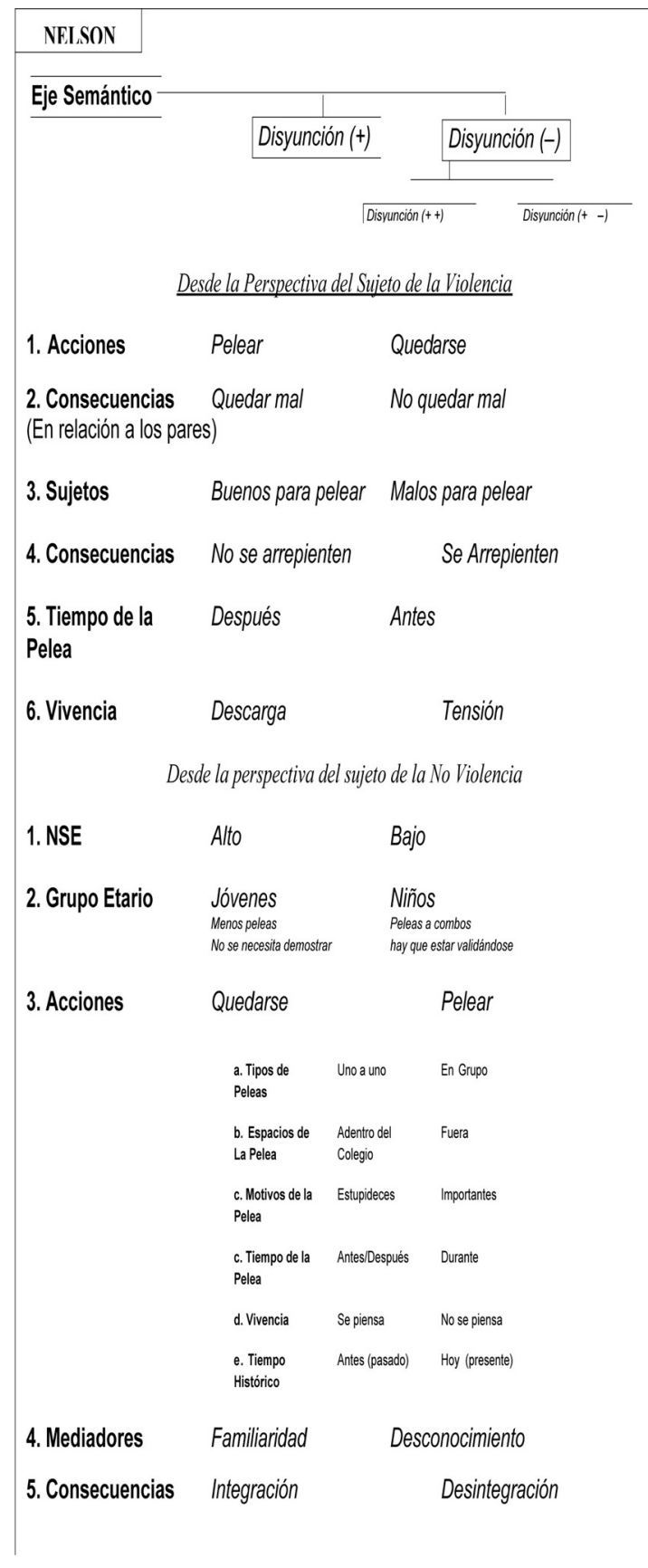

\section{a) Paráfrasis de la estructura del} discurso de Pedro

La aspiración última de pedro, la relación a Si mismo que quiere constituir es ser un sujeto reconocido y libre. El reconocimiento lo obtiene tanto con el respeto de los otros como con su temor. Por el contrario, lo que quiere evitar ser, es ser alguien humillado y preso, es decir alguien que es pasado a llevar por los otros. De ahí que la acción positiva sea el "pelear", pues permite evitar se dominado u humillado por los otros. Por el contrario la acción de quedarse, es decir ceder ante las provocaciones y renunciar a pelear, conduciría a constituir el si mismo negativo. Por ejemplo nos dice "... no se van a rendir por tener miedo. O sea una vez que ya estay metido es imposible salirse porque si te sales te discriminan después y te dicen "ahh, ese es maricón".

Nótese la siguiente paradoja: Ser libre implica para Pedro la indiferencia de los otros, que no lo molesten, que no se metan con él. Y al mismo tiempo aspira a cierto reconocimiento de estos. ¿Cómo se puede ser reconocido por pares indiferentes?

Los medios para desarrollar las acciones son en el discurso de Pedro distintos tipos de colegios y sistemas normativos. Los medios positivos, los que facilitan el despliegue de las acciones positivas la constitución del sí mismo tambien positivo, son los "colegios liberales/flexibles" que tienen normas no formales. Por el contrario, los medios negativos serían los "colegios estrictos" que funcionan con normas formales e inflexibles, a saber, prohibiciones o limitaciones referidas a la forma de la imagen y de la conducta. Y no a lo esencial. Por ejemplo Pedro nos dice: "O sea más que estrictas yo creo que son muy anticuadas, además hay algunas que son como tontas, o sea hay colegios en que te limitan todo (...) o sea no te vay a sacar un siete o no te va a ir mejor porque anday con el pelo corto o largo".

En la lógica de su discurso, lo colegios tradicionales, por la imposición de normas for- 
males, controlan la conducta de los estudiantes, de modo que estos se "quedan" pero a costa de vivir un stress, incomodidad y presión, por el hecho mismo de no poder descargarse, que tendría como efecto ulterior la explosiones agresivas. Por el contrario, los colegios modernos y liberales garantizan una vivencia de mayor bienestar pero no garantizan la ausencia de peleas, sino que las disminuyen. De algún modo, en este discurso los colegios tradicionales no tendrían éxito en el control de la agresión y la pelea. En relación a esto último nos cuenta: "De repente creo que en un colegio pueden haber más peleas por el estrés que traen los alumnos de tener que andar todo el día a la orden de los profesores (...). A veces cosas chicas, como tener que estar desde las 8:30 hasta las 4:00 te irrita, o sea son cosas chicas pero que a la larga influye".

Para Pedro entonces la violencia en la escuela, y particularmente las peleas entre pares, responden a una lucha de prestigio por el reconocimiento y a una búsqueda de libertad. Es desde esta búsqueda de la constitución del sí mismo que el pelear no es una acción negativa, sino por el contrario, deseable y necesaria. Ciñéndonos al discurso de Pedro, pareciera que la ambigüedad de la búsqueda en cuanto a la constitución del sí mismo tiene que ver con que quiere ser libre respecto de las normas y la institución escolar y quiere ser reconocido por los pares. De este modo, es posible considerar que los destinatarios de las dos aspiraciones identitarias son distintos.

Por último, el eje semántico "Vivencias del sujeto", indica que la acción "pelea" se asocia a una vivencia de satisfacción entendida como descarga "E: ¿Y qué se siente después de la pelea? P: Se siente descarga y satisfacción". En cierta forma podría decirse que la descarga es descarga de la Humillación, de la burla. Es deshacerse de esos significados que constituyen el Sí mismo negativo. Por lo tanto se busca la descarga de un significado, lo que entraña un paradoja. Usa una representación energética para un problema de significado. b) Paráfrasis de la estructura del discurso de David

De lo que se trata en el proyecto de David, es de Ser libre, lo que implica aceptarse y quererse a sí mismo, que sería la condición que permite no vivir aproblemado y poder surgir en la vida. La acción interior necesaria para alcanzar esta modalidad positiva del Ser, es descargar la Energía negativa, que es la violencia, la cual sería natural en todo ser humano, y por lo tanto es inevitable el tener que hacer algo con ella. David nos dice: "Depende de si la reprimes o no la reprimes. Mientras más la reprimes, después se expresa más en destrucción". Expresarla, no reprimirla, sería la acción positiva que posibilita el si mismo positivo. Pero es un modo de "estar en paz consigo mismo y en guerra con los demás", es decir que hay un conflicto inevitable con el otro al constituir el Sí mismo positivo. Sin embargo, la expresión agresiva, generarían menos violencia, y menos destrucción. La represión de la violencia natural, generaría una violencia interna, problemas, conflicto, ira, proyección en los otros, y en ultimo termino una "explosión" con consecuencias mucho más destructivas: "Igual la persona explota. Todos al final nos ponemos violentos".

Paradojalmente entonces, en esta lógica, el que reprime la violencia es potencialmente más destructivo, puesto que no se acepta, vive de algún modo "poseído" por la energía negativa, la conserva, no la tramita, y llega a la explosión, o la ejerce contra sí mismo. Es una constitución del sí mismo negativo que en última instancia conduce a la sobreadaptación, que en la estructura simbólica del discurso de David se traduce como muerte.

También paradojalmente, la expresión de esta violencia natural, es un modo de culturizarla, de instalarla en el lazo social, y de al menos interesarse por lo otros. Contrariamente, el reprimido está tan concernido por sí mismo, que es "indiferente a los demás" o los discrimina, proyecta en ellos su negatividad constituyente. He aquí algunos textos de David: 
En el fondo, el tratar de no tener conciencia de eso [violencia], porque es penca cachar que uno también es mierda, que uno no es tan bueno como quiere ser, y tirai', proyectai' todo eso sobre la demás gente. Pero se proyecta como violencia, como no aceptación de los demás"

"Porque en el fondo, si estas metido en esa cuestión tuya [energía negativa, violencia natural] tampoco puedes surgir. Ahí eso lo expresas como violencia y asi puedes vivir".

Los agentes inspiradores de las acciones positivas, están representados por la figura del "Flaite", joven de NSB, no reprimido, "punga", marginal, pero que se quiere a si mismo, se acepta, porque expresa su violencia y no se reprime:

"E: ¿Y tu cachai locos que anden armados? / D: Si, hay unos locos que son terrible de tiraos a flaite./ E: ¿Y por que tu creis que andan armados? / D: Es la moda, es la onda. / E: Es una onda no más, o.../ D: Es darsela de flaite en el fondo... darsela de flaite. En este colegio esta de moda darsela de Flaite".
Los agentes negativos, están representados por la figura del "cuico", sobreadaptado a las exigencias sociales, y por lo tanto reprimido, incapaz de expresar su violencia pero potencialmente destructor en el momento de la explosión. Es muy interesante observar está estigmatización ambivalente de la figura del Flaite: es una figura marginal, y por tanto discriminada, pero al mismo tiempo admirado por su libertad y su expresividad. Se trata de una suerte de identificación al Flaite, pues es el icono de la acción de la descarga de la energía negativa.

\section{Estructura Común}

Presentamos aquí un modelo que refleja lo que de común hay en los distintos casos. El énfasis esta puesto entonces en la estructura general del discurso, más allá de los matices particulares y singulares (ver Figura 3). Este nivel de análisis si bien pierde en singularidad, gana en la posibilidad de construir un modelo que de cuenta en parte de la lógica cultural que opera en el fenómeno de la violencia escolar en el nivel socioeconómico alto.

Figura 3. Estructura Integrada

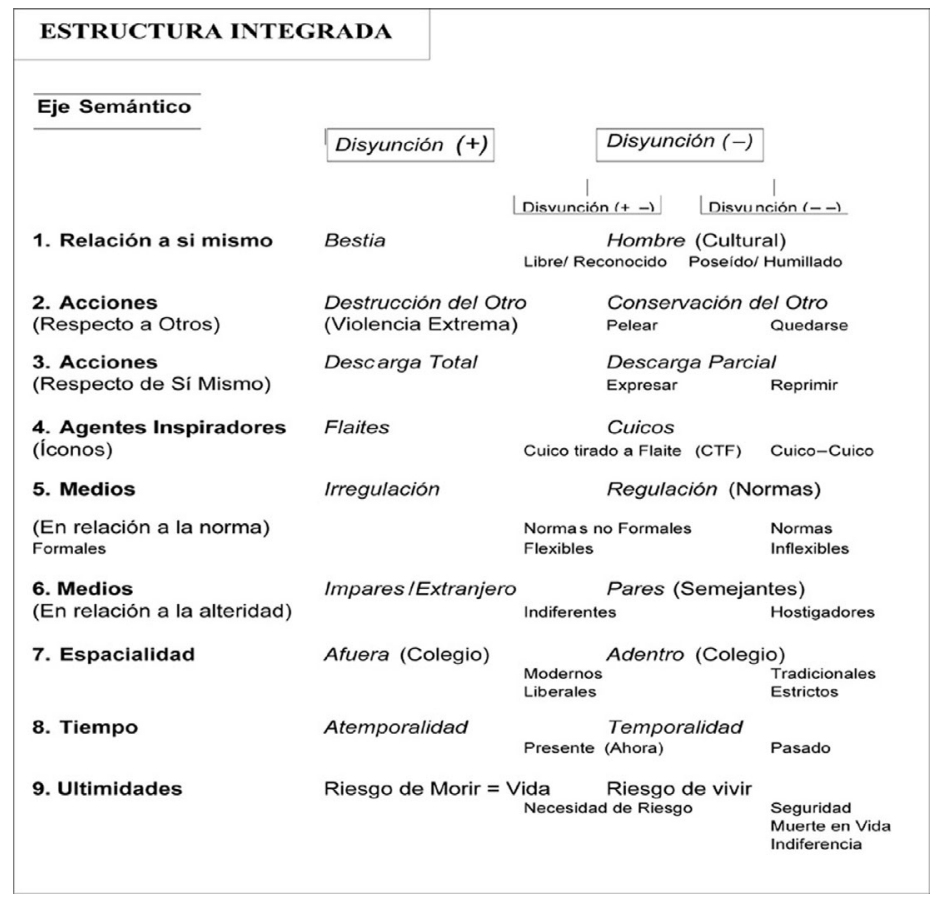




\section{Paráfrasis de la estructura común}

La estructura general del discurso de los entrevistados, es una estructura jerarquizada, pero dentro de la cual hay relación de implicación a dos niveles. Los ejes semánticos principales se implican mutuamente entre sí y también los ejes semánticos secundarios, que en el fondo constituyen un desglose del polo negativo de la secuencia principal.

Es interesante notar que el material desglosa el polo negativo y no el positivo. La razón de esto es que el locutor del discurso que privilegiamos en el análisis es la de "Cuico tirado a Flaite", pues es esta figura la que encarna la violencia en el NSA, que es el objeto de este artículo. Si se privilegiara como locutor el "Cuico-Cuico" los ejes semánticos serían practicamente los mismos pero se invertirían las valoraciones.

\section{a) Paráfrasis de planos semánticos principales}

Todo discurso social contiene formalmente elementos que dan algún sentido a la vida, que organizan una visión coherente del mundo y de la existencia humana, que entregan o sugieren medios para la integración de la conducta cotidiana y que sitúan el ser en una perspectiva de transformación hacia un deber ser. Esto último implica que el discurso social suele situar al sujeto en una cierta ambigüedad o ambivalencia puesto que estando constituido por una negatividad, ella es sin embargo trabajable, transformable (Hiernaux, 1995)

En el discurso sobre la violencia de los jóvenes escolares agresivos de nivel socioeconómico alto, aparece que lo que quieren ser (polo +) es ser un hombre "natural", no sujeto a las convenciones ni a la regulación social de sus tendencias, lo que se sintetiza bien en la figura de la bestia "Si, yo creo que es parte de la naturaleza humana. O sea, de hecho en las manadas de lobos también esta, esta en todos lados" "es un instinto violento -nos dicen-, igual somos animales, somos animales, somos bestias. Simul- táneamente, lo que no quisieran (polo -) es ser convencionales, sujetos domesticados y plegados a la norma y en ultima instancia la cultura. La imagen del "hombre cultural", esta investida en el discurso de la más radical falta de espontaneidad, de una ignorancia de si mismo, de sus instintos, de las pasiones y energías que lo atraviesan: "Porque no se sienten bien... porque no nos sentimos bien". Ahora bien, el sujeto social cuyo discurso analizamos, se presenta a sí mismo como "cuico tirado a flaite", lo que implica que la aspiración a constituir el ser y la identidad en los términos de una bestia natural es sólo una aspiración, pues de hecho es un hombre regulado y cultural. Y desde ahí, estos sujetos buscan al menos ser "hombres reconocidos y libres" y no sujetos incapaces de liberar sus instintos (poseídos) ni humillados por los otros. La búsqueda de libertad y reconocimiento para constituir el ser y la identidad, es un modo de acercarse a la naturalidad (bestial) sin encontrarla nunca plenamente. Pero al menos evitaría ser un sujeto poseído y humillado cuyo icono sería la figura del "cuico-cuico", es decir el joven de Nivel socioeconómico alto que no intenta salir de su condición de extrema regulación social y cultural.

Las acciones necesarias para alcanzar el polo positivo del ser serían, entre otras, la violencia extrema hacia los otros, que implicaría una "descarga total" de la energía negativa. Descarga total implica ausencia total de control y de regulación, que daría lugar, en ultima instancia, a la destrucción del otro. Por eso constituye al ser bestial. Por el contrario, las acciones que constituirían el ser negativo, serían formas de descarga parcial, que posibilitan, por su limitación, la conservación del otro. Se observa en la estructura que la descarga parcial toma a su vez dos formas: la expresión o la represión: "Depende de si la reprimis o no la reprimis. Mientras más la reprimis, después se expresa más en destrucción".

Los medios que permiten realizar las accione positivas, son la irregulación, la ausencia de normas, lo que permitiría la descarga 
ilimitada, extrema, de todas las pulsiones y energías, incluida la violencia. Las normas serían por su parte los medios negativos, que solo posibilitan una descarga parcial y por tanto implican una constitución de sí mismo no natural (Hombre cultural).

Los agentes inspiradores, son los agentes o íconos, que inspiran o ponen a disposición del sujeto los medios y acciones para constituirse positiva o negativamente. El Flaite es el agente inspirador positivo. Se trata de un joven marginal, provocador, transgresor, frecuentemente armado y que inspira temor, respeto, y hasta una cierta admiración. Su provocación y agresión insistente sería "justificada" según nuestros informantes, pues los flaites tendrían motivos de sobra para provocar y agredir. El agente inspirador negativo es el "cuico", quien inspira las normas, la regulación, la represión, hasta el sometimiento.

Una cuestión aparentemente secundaria, pero muy importante, es la construcción del espacio que tienen los jóvenes. En el modelo observamos que opera un disyunción clásica: Afuera y adentro, siendo el adentro la escuela, la escuela del cuico-tirado a Flaite. El Flaite estaría afuera, en la escena social, en las calles, en lo barrios pobres. Se lo encuentra rara vez, o se lo ve en los medios. Por lo tanto el espacio de la irregulación, del exceso natural y bestial es el afuera, lo que va ligado a temores y una serie de aprehensiones respecto a lo que ocurre fuera de la escuela, fuera de la comuna, etc. El adentro, la escuela, es entonces para ellos el espacio normado, y seguro, pero que imposibilita la constitución positiva de sí. Vemos como se organiza una relación ambivalente al afuera y al Flaite. Este es admirado, situado incluso como ideal, pero es temido, lo mismo que el espacio externo.

En cuanto al tiempo, observemos que aparece un resultado enigmático. El Flaite, como icono de la bestia y la irregulación, es situado atemporalmente. No es de ayer, no de hoy, está fuera del tiempo, lo que sugiere que se trata de una verdadera figura mitológica urbana.
En la temporalidad están el sujeto que habla en esta estructura, el cuico tirado a Flaite y también el cuico. La aspiración a ser un Flaite va de la mano con la aspiración-ilusión de salir del tiempo, de existir ahistóricamente.

Finalmente, nos ha parecido importante reflejar en la estructura lo que está en juego en última instancia para el sujeto de este discurso. Es lo que llamamos ultimidades, siguiendo a Hierneaux (1995). Lo que muestra el modelo es que estos jóvenes quieren vivir, sentir la vida plenamente y con libertad, pero esa aspiración última implica, de acuerdo al icono del Flaite, estar en riego de morir, de atravesar riegos de tal magnitud que ponen en riesgo la propia vida. Eso es lo temido, pero también lo admirado. Desde la admiración la vida esta en el riesgo de morir, mientras que la ultimidad negativa sería el riesgo de vivir, que se desglosa en dos polaridades: la necesidad de riesgo y la búsqueda extrema de seguridad. El punto central que emerge aquí, es que para vivir, para vivir con sentido, habría que buscar el riesgo, lo que da cuenta de un desafío implícito a la creciente ideología de la seguridad.

\section{b) Paráfrasis de los planos semánticos secundarios}

Si observamos con detenimiento la estructura paralela integrada de la información, podemos darnos cuenta que desde el polo negativo de cada disyunción emerge jerárquicamente otra estructura paralela -en este caso secundaria-, que refleja ya no tanto la relación que el "Cuico" establece hacia el "Flaite" en dialéctica con él, sino más bien la relación y diferenciación que se establece al interior de su propio grupo social.

Si ubicamos esta estructura paralela secundaría en una sola línea como aparece en la figura anterior, podemos ver que cada una de estas disyunciones secundarias mantienen cierta relación entre sí. De ahí la relevancia de realizar este análisis de segundo orden. 
Sin embargo este análisis secundario de la estructura, en tanto refleja el lugar de la violencia de este nivel socioeconómico, se instala como el foco principal desde donde se construye la perspectiva del discurso de estos jóvenes. De esta manera, la relación del "Cuico" al "Flaite", es relevante en tanto el Flaite ocupa ese lugar lejano, extraño y polar, y al mismo tiempo, el lugar de una posición idealizada sobre el hecho de su supuesta libertad. Por el contrario, el verdadero drama se urde al interior del propio nivel socioeconómico, donde el "Cuico", cuestiona su posición a través de la figura ambigua del "Cuico tirado a Flaite". Este último personaje alude a aquel que siendo de nivel socioeconómico alto toma las características usuales del icono del nivel socioeconómico bajo, el Flaite. De esta forma, el "Cuico tirado a Flaite" (CTF, de ahora en adelante), tiende a vestirse a la usanza del hip-hop, se comporta y habla de un modo similar al "Flaite", usa en cierta forma los códigos y claves lingüísticos que surgen desde éste último y en algunas ocasiones llega a usar armas: "en muy pocos casos los que son como uno pero se las dan de "rotos" y ellos andan con cuchillos". De algún modo, en el análisis de esta estructura paralela secundaria este CTF, alude a aquel "Cuico" que, sintiéndose desagradado en su lugar de "Cuico", busca otro camino que por una parte le otorgue el sentido perdido a su posición y que por otra, le permita encontrar la vía para liberarse de las amarras normativas, por las cuales se siente reprimido y asfixiado.
Figura 4. Estructura Secundaria de la Jerarquía

\begin{tabular}{|c|c|c|}
\hline EJE SECUNDARIO & & \\
\hline Eje Semántico & & \\
\hline & \begin{tabular}{|l|} 
Disyunción (+) \\
\end{tabular} & $\frac{\mid}{\text { Disyunción (-) }}$ \\
\hline $\begin{array}{l}\text { (Relación a si mismo) } \\
\text { Hombre (Cultural) }\end{array}$ & Libre/ Reconocido & Poseído/ Humillado \\
\hline $\begin{array}{l}\text { (Acciones) } \\
\text { Conservación del Otro }\end{array}$ & Pelear & Quedarse \\
\hline $\begin{array}{l}\text { (Acciones) } \\
\text { Descarga Parcial }\end{array}$ & Expresar & Reprimir \\
\hline $\begin{array}{l}\text { (Agentes) } \\
\text { Cuicos }\end{array}$ & $\begin{array}{l}\text { Cuico tirado a Flaite } \\
\text { (CTF) }\end{array}$ & Cuico-Cuico \\
\hline $\begin{array}{l}\text { (Medios) } \\
\text { Regulación (Normas) }\end{array}$ & $\begin{array}{l}\text { Normas no Formales } \\
\text { Flexibles }\end{array}$ & $\begin{array}{l}\text { Normas Formales } \\
\text { Inflexibles }\end{array}$ \\
\hline $\begin{array}{l}\text { (Medios) } \\
\text { Pares }\end{array}$ & Indiferentes & Hostigadores \\
\hline $\begin{array}{l}\text { (Espacios) } \\
\text { Adentro (Colegio) }\end{array}$ & $\begin{array}{l}\text { Modernos } \\
\text { Liberales }\end{array}$ & $\begin{array}{l}\text { Tradicionales } \\
\text { Estrictos }\end{array}$ \\
\hline $\begin{array}{l}\text { (Tiempo) } \\
\text { Temporalidad }\end{array}$ & Presente & Pasado \\
\hline $\begin{array}{l}\text { (Ultimidades) } \\
\text { Riesgo de vivir }\end{array}$ & Necesidad de Riesgo & $\begin{array}{l}\text { Seguridad } \\
\text { Muerte en Vida } \\
\text { Indiferencia }\end{array}$ \\
\hline
\end{tabular}

Sin embargo, este CTF es un pretendiente por excelencia por una parte y por otra, ocupa el lugar -ambiguo por cierto-, de aquel que siendo hijo de extranjeros nace en un país del cual no es ni se siente parte. Por tanto su condición, es la condición de un insatisfecho, que encubre el drama imposible de su deseo: ser reconocido y al mismo tiempo, libre. Ambos puntos que en la práctica, se muestran inconectables. De esta forma el CTF, extrañamente necesita de pares indiferentes pero que al mismo tiempo lo reconozcan.

La posición doblemente negativa -aquella del Cuico-Cuico- refleja no solo el lugar de un personaje reprimido, sino también el lugar de un poseído, de un esclavo de la norma, que es incapaz de expresarse y que a través de este mismo carácter, es incapaz de hacer frente a la humillación que implica el hostigamiento del otro: "Entonces se lo guarda, se guarda todo su ira, y al final, es como más, una violencia más interna. Entonces queda más dentro de ti que con la demás gente. Como que estai en paz con los demás, pero en guerra contigo mismo".

El medio positivo que permite que el CTF pueda realizarse, es la norma flexible que se 
resuelve en la no formalidad. Es decir, la norma que aún siendo un límite y una frontera, permite un rango de acción posible: "lo importante es que [los profesores] tengan buena onda con los alumnos, ni tan estrictos ni tan relajados, porque hay algunos que no ponen anotaciones negativas y no retan por falta de carácter más que por "buena onda". También hay algunos que no te dejan hacer nada y también es charcha [malo]". Por el contrario, las normas estrictas y formales son medios que lo acercan al polo doblemente negativo de su ser. Son normas que al centrarse en lo visual-formal -es decir en un ámbito que queda anclado en la imagen, desconocen su verdadera constitución para relegarle a lo superficial (normas sobre limpieza, corte de pelo adecuado, uso o no de aros, etc.): "A este colegio igual le cambiaría todo el rollo que hay con el uniforme y tener que venir con pelo corto. En mi opinión acá nos tienen bien reprimidos, no nos dejan hacer nada". Este operar de la norma sería característico de los colegios tradicionales, de ahí que se vea a estos como oponentes a la constitución de lo que quieren de sí mismos.

De esta manera se puede decir que los estudiantes de NSE alto, establecen una relación mucho más estrecha a la norma de lo que aparentemente muestra el discurso de los de NSE bajo. Es esta, la que establece los puntos de referencia desde donde se entiende toda la realidad: lo demás, lo que parece escapar a la norma se desconoce, se teme. El "Flaite" como irregulado se admira y se teme al mismo tiempo: "...es que uno tiene que cachar con quien se está metiendo y si son gallos que vienen como de la población [NSE bajo] uno no sabe qué pueden sacar, o sea sabe que pueden sacar armas pero no sabe qué", dice uno de nuestros informantes.

Por otro lado, el impedimento de la norma que regula, hace que su descarga, como acción fundamental a la que aspiran, sea irremediablemente parcial: solo les quedan dos opciones, expresarla en la medida que se lo permite la norma o reprimirla. Ninguna de ellas es perfecta, no obstante, una es peor que la otra. Reprimirla en última instancia significa quedarse en las amarras de la norma, verse subyugado y asfixiado por ella y lo que es peor, quedarse en definitiva con esa energía negativa y destructiva dentro de sí: llevar la muerte a cuestas "Entonces se lo guarda, se guarda todo su ira, y al final, es como más, una violencia más interna" "Mientras más la reprimis, después se expresa más en destrucción". La única posibilidad por tanto, deriva de la expresión parcial de ésta. En este caso, la pelea, que permite la liberación de este dominio destructivo o como nos dice un informante "en guerra con los demás pero en paz con uno mismo". Lo interesante de la pelea, es que tampoco escapa a la norma, en tanto que aún siendo enormemente violenta permite la conservación del otro. El Flaite como vimos antes, desde este discurso, parece instalarse desde la destrucción del otro. La pelea por el contrario, esta regulada por códigos estrictos que permiten ciertas acciones y no otras. Aquel que no expresa y se reprime, es el mismo que se "queda"6 a fin de cuentas. De ahí, que ante todo, la acción fundamental es no "quedarse" ante el hostigamiento del otro. El que reprime tiene pares que lo hostigan y de los cuales no puede zafarse; por el contrario aquel que se expresa y pelea, logra no ser molestado, no ser tocado (pares indiferentes). De esta forma, se entiende que el sujeto reprimido sea presentado como un sujeto humillado o que acepta ser humillado y que al mismo tiempo, está poseído: "Porque es el 'paiplon' [sin carácter], siempre hay alguien al que huevean... le cuesta expresarse, entonces... la gente también aprovecha de ir a descargar la violencia con el que no se la va a devolver, porque no sabe cómo".

6 En el uso de estos informantes "quedarse" implica no querer pelear o tener miedo de ello y por tanto no hacerlo. 
Colegios y normas que ayuden a la represión de sí, siguen esta misma línea de coacción del si mismo y de esta forma, quedan más cargados de un halo negativo.

La represión instala una particular relación al tiempo: el recuerdo. Aquel que reprime esta sujeto a cierta temporalidad que lo liga irremediablemente a un pasado, un pasado que lo significa. Si analizamos en literal la palabra "quedarse", implica en cierta forma un quedarse con algo, un seguir arrastrando desde el pasado. Por el contrario, uno podría decir, que la pretensión de estos informantes es todo lo contrario: el olvido. De ahí que la relación al tiempo del CTF se vea desde el presente. La acción actual, la pelea por ejemplo, es en cierta forma un olvido de si "cuando me pongo a pelear no se nada de mí". "Es que durante la pelea uno no piensa en nada, estay dándole no más...". Todo lo ligado al arrepentimiento y la culpa -elementos que incluyen en si una relación al pasado- escapan completamente a su pretensión: "no me arrepiento, nunca me arrepiento".

La última disyunción de la estructura que presentamos son las ultimidades, eje semántico que lo debemos a Hiernaux (1995) y que da cuenta de lo que está en juego en última instancia para el sujeto. Las ultimidades que pueden preverse en este caso quedan claras en relación a la disyunción positivo/negativo. Sin embargo al entrar en la jerarquía paralela del polo negativo la situación se nos complica. La vida del "Cuico" -esta sería la interpretación-, al estar marcada por una normatividad que oprime y humilla, está montada por un rango de acción extremadamente limitado que deja poco espacio a la espontaneidad y que por lo mismo, tiende hacia la asfixia de la movilidad vital. La contradicción en este punto es clara: la seguridad que ostenta el "Cuico" en su vida (contraria al riesgo permanente del Flaite), va de la mano de una imposibilidad de ser ellos mismos (la idea de libertad). De esta forma, la posición doblemente negativa en la cual se encumbra el Cuico, es decir la figura del Cuico-Cuico, ampara del mismo modo una condición extraña y de alguna manera perversa para estos jóvenes: el de estar muerto en vida. La inmovilidad vital es tal, que en cierta forma el destino tiene una certeza angustiante, frente a la que el CTF se rebela. Estar muerto en vida apunta a que la vida del cuico no entraña ninguna incertidumbre, ningún cambio inesperado, ninguna crisis relevante. El cuico de esta forma es representado en el discurso como un muerto vivo, un zombie caminante sobre una vida que no es vital. Este estar muerto en vida tiene una consecuencia contradictoria también: la indeferencia. Ya no es solamente la necesidad del CTF de querer pares indiferentes, sino también la visión que tienen estos jóvenes de que a fin de cuentas el Cuico-Cuico es indiferente: no le interesa el otro. Esto solo puede entenderse si entiende la oposición positivo/negativa desde la cual surge. El Flaite al estar en riesgo de morir renueva constantemente el sentido de su vida y no solo eso, sino que en el hecho mismo de su riesgo renueva el interés en el otro solo que de un modo radical. En el hecho de violentarse el Flaite parece renovar el pacto social al derrumbar su natural tendencia a la estabilidad y el orden: hace de la vida un corruptible y de esta forma, una posibilidad de seguir haciendo. Al reprimir su vitalidad el Cuico-Cuico es potencialmente más destructivo. Es una constitución del sí mismo doblemente negativo que en última instancia conduce a un control rígido de si mismo, a una suerte de sobreadaptación, que en la estructura simbólica de estos jóvenes se traduce en muerte.. Para que haya algo de violencia es necesario cierto interés por el alter; no expresar esta violencia (guardársela, reprimirla), puede lograrse a costa del que el otro me sea indiferente. La contradicción entonces es la siguiente: a favor del pacto social el Cuico-Cuico reprime su vitalidad y de esta forma, se ve poseído por la norma y humillado en ella, sin embargo el costo de esta renuncia por el pacto social, es que el otro no me cause ningún interés. Por el con- 
trario, el Flaite en la búsqueda de la destrucción del otro hace de éste un reconocido por excelencia, lo que a fin de cuentas instituye un fuerte lazo social.

La posición que asume en este caso el CTF, es una interrogante difícil de contestar. Sin embargo al acercarla y contraponerla a su oponente (Cuico-Cuico), la posición de seguridad de éste devela lo que está en juego en última instancia para el CTF: la necesidad de riesgo. La seguridad del Cuico-Cuico certeza que acerca a la forma de la muerte-, denuncia la inclinación del CTF de ponerse en riesgo "a propósito", como un modo de contrarrestar la asfixia normativa que sufre. El CTF se retuerce ante la posibilidad cierta de que esto ocurra y rompe el circuito de su certeza a través de la violencia, poniendo al riesgo -así como el Flaite-, en el centro de su hambre de vitalidad y sentido.

\section{Conclusión}

¿Qué nos ha permitido extraer este análisis? Ante todo, la estructura del discurso hace aparecer un código implícito a la violencia escolar en el nivel socioeconómico alto, código que no es inmediatamente perceptible en la escucha de los informantes o a la lectura del material de entrevistas. Este código tiene el interés de condensar los valores, preferencias y juicios socialmente determinados, y de los cuales los jóvenes en cuestión son portadores, particularmente los jóvenes que recurren a la violencia, la agresión y las peleas. En este sentido, las estructura representa la cultura interiorizada (Hiernaux) de los"cuicos tirados a flaite". No se trata entonces de la "cultura objetivada" (Bourdieu), que se refiere al nivel de aprehensión de la cultura a través de las huellas sensibles que dan cuenta de modos de ver, pensar y sentir, tales como las modas vestimentarias, la siñalética en las calles, los estilos constructivos, las tecnologías usadas, etc. Tampoco se trata de la "cultura en sí" (Bourdieu) que se refiere al código abstracto, en tanto sistema organizador de la vida. El nivel de la "cultura interiorizada" aperece como intermediario, cordinando el código a las acciones, y tiene claramente funciones sociales de compensación y legitimación (de la violencia y la agresión en este caso), pero también funciones psicoafectivas de integración de la conducta cotidiana (lo que reduce la tensión a nivel subjetivo) y de movilización afectiva, ubicando al sujeto en una búsqueda de una relación a si mismo (lo que implica un aumento de la tensión subjetiva).

De esta manera, nuestro análisis profundiza la sugerencia hecha por Ortega y MoraMerchán (2000), quienes sostienen que la violencia interpersonal en la escuela es un fenómeno más cultural que natural, ya que el comportamiento agresivo ha perdido su justificación biológica y los actores no buscan con él un beneficio de mantenimiento de la vida o de lucha por la supervivencia. En efecto, la estructura presentada nos muestra a todas luces que lo que se busca es algo simbólico, la libertad respecto de si mismo, de la propia interioridad, y el reconocimiento de los otros, de los pares.

La estructura muestra que estos estudiantes "agresores" en el seno de la escuela, representan el sentido de su recurso a la violencia como una acción articulada a una cierta busqueda de como les gustaría ser, a ciertos agentes y medios que les permiten identificar las acciones a seguir, y el conjunto se entrama en una cierta relacion al tiempo y al espacio, cuestiones decicivas a resolver para todo código cultural.

Más allá del interes teórico que los resultados tienen, nos parece que hay aportes para la intervención en el sentido de mostrarnos a qué discursos estos actores pueden ser sensibles y a cuales no. Para mostrar esto examinese el esquema siguiente (figura 5). 
Figura 5: Cruces de planos semánticos dela estructura secundaria

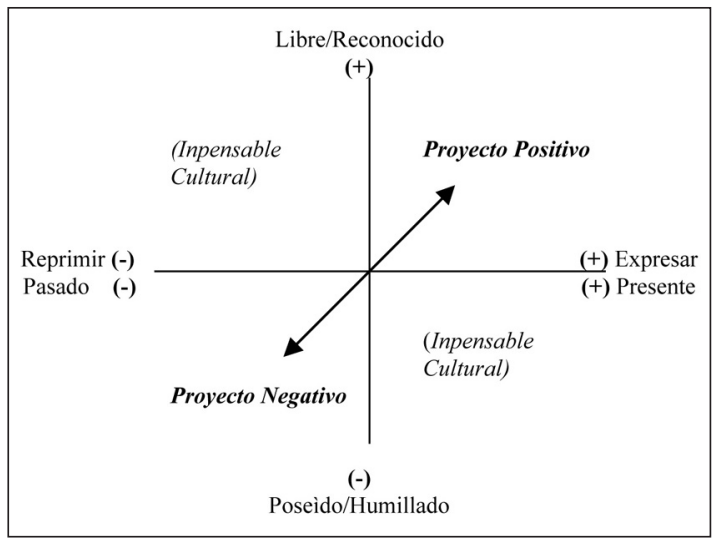

Se cruza el plano semántico de la Relación a si mismo (eje vertical) con los planos de las acciones respecto de si y el tiempo (eje horizontal) obtenidos en la estructura secundaria. Asi, vemos aparecer que el proyecto positivo del discurso que hemos analizado, implica que mientras más se acerca el sujeto a la expresión de sus pasiones y energías, y mientras más se situa en relación a referencias temporales presentes y actuales, más chances tendrá de devenir un sujeto libre y reconocido, que es lo buscado fundamentalmente. Si agregaramos los otros ejes, obtenemos que tambien hay más chances de devenir libre y reconocido al pelear y responder a las provocaciones, al estudiar en un colegio "moderno", con normas flexibles y no formales, en los cuales sería posible encontrar pares indiferentes que no hostigan ni se "meten" en los asuntos de los otros.

Inversamente, el proyecto negativo implica que mientras más el sujeto se "queda" (y no responde a las provocaciones), mientras más reprime sus impulsos agresivos y existe refiriendose al pasado, más deviene poseido y humillado. Del mismo modo, si tomamos el ultimo plano de la estructura, la lógica del proyecto negativo es que mientras mas seguridad se instala en la vida y en la escuela, mas carente de sentido se torna el vivir. Es por eso que la necesidad de riesgo es parte central del proyecto positivo, como si solo al haber riesgo la vida pudiera tener interés y sentido, permitiendo la constitucion de un sujeto libre y reconocido.

Ahora bien, hay dos cuadrantes del esquema que situan "inpensables culturales" para los jóvenes del discurso analizado.Esto quiere decir que para estos jóvenes es inpensable que "quedándose" o controlándose al momento de ser provocado se pueda ser libre y reconocido por los pares. No es solo que no esten de acuerdo con esta idea, sino que ella es iconcebible desde la lógica discursiva que los anima. Del mismo modo, resulta inpensable que en el pasado se halla podido ser reconocido o libre, ni tampoco en instituciones o modos de vida seguros. De tal suerte, discursos de prevención o intervención que van en la dirección de una "mayor y mejor seguridad social", o aquellos que alertan contra las influencias negativas del presente, la tecnología, la información u otros dominios, son probablemente inteligibles e importantes para los adultos o estudiantes no agresivos, pero son ininteligibles, puesto que impensables desde la cultura interiorizada, para los jóvenes agresores de nivel socioeconómico alto. Pensamos que para la prevención y la intervención sobre el fenómeno de la "violencia escolar" debe tenerse en cuenta estas lógicas para poder interpelar a los jóvenes. No se trata de naturalizar sus discursos, ni de avalarlos, sino de captar su logica para poder dialogar con ellos evitando, en lo posible, de caer en el repetitivo dialogo de sordos.

\section{Referencias}

Abramovay, M. (2003) Enfrentando a Violencia nas Escolas: um informe do Brasil. En Violência Na Escola. América Latina e Caribe. Brasilia: UNESCO.

Aron, A. (2000) Un programa de educación para la No-Violencia. Revista Psykhe, 2 (9), 25-39. 
Bordieu, P. (1977) La reproducción: Elementos para una Teoría del Sistema de Enseñanza. Barcelona: Editorial Labor.

Clastres, P. (2004) Arqueología de la Violencia. La guerra en las Sociedades Primitivas. Mexico D.F: Fondo de Cultura Económica.

Contador, M. (2001) Percepción de Violencia Escolar en Estudiantes de Enseñanza Media. Revista Psykhe, 1(10), 69-80.

Defensor del Pueblo (1999) Informe del Defensor del Pueblo sobre Violencia Escolar. Madrid.

Dubet, F. \& Martuccelli, D. (1998) En la Escuela: Sociología de la Experiencia Escolar. Buenos Aires: Losada

FiLmus, D. (2003) Enfrentando la violencia en las Escuelas: Un informe de Argentina. En Violência Na Escola. América Latina e Caribe. Brasilia: UNESCO.

Foucault, M. (2001) Vigilar y Castigar. México D.F.: Siglo Veintiuno Editores.

García, M. \& Madriaza, P. (2004b, en prensa) Sentido y Sinsentido de la violencia escolar. Análisis Cualitativo del Discurso de Estudiantes Chilenos. Revista Psykhe. Escuela de Psicología P.U.C.

García, M. \& Madriaza, P. (2004a) Clamores Juveniles y su Relación con la Violencia Escolar: Conflicto y Aspiración por Recrear un Orden Social. Revista Persona y Sociedad 18(1). Pp. 31-50.

GeERTZ, C. (1986) “Descripción Densa: Hacia una Teoría Interpretativa de la Cultura" en La Interpretación de las Culturas. Editorial Gedisa: Barcelona.

GIRARD, R. (1983) La violencia y lo sagrado. Editorial Anagrama: Barcelona

Hiernaux, P. (1995) Analyse estructurale de contenus et Modèles culturels en Al-Barelo
L., de Saint Georges P., Digneffe F., Hiernaux J-P., Maroy Ch., Ruquoy D. (1995) Pratiques de recherche en sciences sociales. Ed. Arman Collin: París

Instituto Nacional DE LA Juventud (2002) Tercera Encuesta nacional de la Juventud. Santiago: Instituto Nacional de la Juventud.

Kaufmann, J. (1997) L'entretient comprehensif. Paris: Nathan.

LipovetZKI, G. (1986) La Era del Vacío: Ensayos sobre el Individualismos Contemporáneo. Barcelona: Editorial Anagrama.

LlaÑA, M. (1999) La perspectiva del Sistema Educacional respecto a la violencia Infanto-Juvenil. Revista de Psicología de la Universidad de Chile, 2(8), 39-43.

Lopez, M. \& Filsecker, M. (2001) Manifestaciones de Violencia y agresividad en la Escuela: estudio comparativo en tres realidades geográficas distintas. Santiago: ICET.

Luhmann, N. \& De Georgi, R. (1990) Teoría de la Sociedad. Editorial Iberoamericana: Mexico.

Magenzo, A. \& Donoso, P. (2000) "Cuando a Uno lo Molestan": Un Acercamiento a la Discriminación en la Escuela. Santiago de Chile: Editorial LOM.

Maluf, N., Cevallos, C. \& Cordoba, R. (2003) Enfrentando la violencia en las Escuelas: Un informe de Ecuador. En Violência $\mathrm{Na}$ Escola. América Latina e Caribe. Brasilia: UNESCO.

Mauss, M. (1995) Essay sur le Don. Forme et Raison de l'échange dans les sociétés archaïques. En Sociologie et Anthopologie. PUF: París

Mena, I. \& Vizcarra, R. (2001) Algo Nuevo bajo el Sol: Hacer visible el Maltrato y Posible la Esperanza. Revista Psykhe, 1(10), 81-101. 
Navarro, L. (2003) Enfrentando la violencia en las escuelas: Un Informe de Chile. En Violência Na Escola. América Latina e Caribe. Brasilia: UNESCO.

Nietzsche, F. (2000) La Genealogía de la Moral. Editorial EDAF: Madrid

O’Donnell, H. (1999) La Violencia en el Sistema Educativo. Buenos Aires: Grupo Editorial Temas.

Olweus, D. (1998) Conductas de Acoso y Amenaza entre Escolares. Madrid: Editorial Morata.

Ortega, R. \& Mora-Merchán, J. (1997) Agresividad y violencia. El problema de la victimización entre escolares. Revista de Educación, 313, 7-27.

Ortega, R. (1998) La convivencia escolar: qué es y cómo abordarla. Sevilla: Junta de Andalucía. Consejería de Educación y Ciencia.

Ortega, R. \& Morta-Merchán, A. (2000) Violencia Escolar: Mito o Realidad, Sevilla, Mergabulm.

Piret, A., Nizet, J. \& Bourgeois, E. (1996) L'alyse Sructurale. Une methode dianalyse de contenu pour les sciences humaines, Bruxelles, De Boeck Université.

Strauss, A. \& Corbin, J. (1994) Grounded Theory methodology. An overview. In Denzin, N. \& Lincoln, Y. (Eds.), Handbook of Qualitative Research (pp. 273-285). Sage Publications.
TAYLOR, S. \& BOGDAN (1986) Introducción a lo Métodos Cualitativos de Investigación. La búsqueda de Significados. Buenos Aires: Editorial Paidos.

USA, Center for Disease Control. (2001). Fact Sheet on Dating Violence. Extraido en Mayo 13, 2003, desde http://www.cdc.gov/ncipc/ dop/yopt/datviol.htm

USA, National Center for Education Statistics (2002) Are America's Schools Safe? Students speak out: 1999 School Crime Supplement. Extraído en Mayo 13, 2003, desde $h t t p: / / n c e s . e d . g o v / p u b s e a r c h$.

VisCARDI, N. (2003) Enfrentando la violencia en las Escuelas: Un informe de Uruguay. En Violência Na Escola. América Latina e Caribe. Brasilia: UNESCO.

WithneY, I. \& SMith, P. (1993) A survey of the nature and extend of bullying in junior/ midle and secondary schools. Educational Research, 35, 3-25.

Wordes, M. \& Nunez, M. (2002) Our Vulnerable Teenagers: Their Victimization, Its Consequences, and Directions for Prevention and Intervention. Washington DC: National Center for Victims of Crime \& National Council on Crime and Delinquency.

ZERÓn, A. (2004, en prensa) Violencia Escolar y Violencia Anti-Escuela: Aportes Teóricos para una Aproximación Sociológica. Boletín de Educación. 
